\title{
Smart Automation: Machine Learning Enabled Workflow for Logic and DRAM
}

\author{
John Flanagan, Ashley Sixtos, Chris Hakala, Justin Roller and Hayley Johanesen
}

Thermo Fisher Scientific, United States

Current S/TEM automation requires highly trained microscopists to build specialized acquisition and analysis recipes for specific semiconductor structures. Recipes can be challenging to write and take a long time, therefore the industry continues to rely on manual operation to overcome TEM analysis demands. Time to develop robust automation is often too slow for the product change rate of industry development cycle. To overcome this barrier, Smart Automation was developed on a Thermo Scientific Metrios ${ }^{\mathrm{TM}}$ AX 200kV S/TEM as an application providing a generalized acquisition workflow that is ready to use without requiring specialist intervention. The application interacts with a Fully Convolutional Neural Network (FCN) through a user-friendly interface for navigation and auto alignments. The FCN is the key enabler for lowering the bar to automation set up with a time to robust automation within hours instead of days.

To facilitate creation of trained FCN models the Smart Automation workflow includes an application called Segmenter, a data annotation and model creation tool (Figure 1). In Segmenter, the user annotates a handful of training images by drawing polygons around features of interest. After a user manually segments the images, the Segmenter software coordinates sending the data to a remote server where the FCN network is built and accessed later at runtime. This allows for customization of the automated data acquisition without the need to re-program the underlying workflow.

There are three key navigational steps in the Smart Automation workflow that require object recognition by the FCN: the lamella shape, the device line, and the individual structures. Variation in lamella shape is common in manual lamella prep. The outcome is influenced by the operator, the lamella prep instrument, and the device type within the lamella. Common template matching techniques include cross correlation and contrast-based edge detection (pattern matching). These methods are partially invariant to linear transformation, occlusion, and lighting, but not morphological variation. In a reliability investigation it was found that classical pattern matching could fail up to $20 \%$ on manually prepared lamella due to morphological variation. By integrating the FCN into the workflow, lamella finding has increased to 100\% robustness in a repetitive use-case test. Similar outcome was observed for the device line (95\%), the line through the thinned window at which devices are located. Individual structure finding routines showed $98.7 \%$ combined for TEM and STEM. Robustness is calculated by the number of identified regions of interest divided by the total number of actual regions of interest. In most cases, the user only needs to segment 60 regions of interest to achieve robust trained models for the individual structures. Time to segment, train the inference server, and validate network to greater than $90 \%$ robustness is achieved in about 2 hours.

In addition to the FCN enhancing workflow navigation, it is also applied in the auto alignment of the Si 110 zone axis in STEM mode. This step is critical for high-resolution semiconductor imaging as the devices are fabricated along principle crystal planes that must be aligned to the incident electron beam. If the final devices are imaged at a tilt $>0.5$ deg from the zone axis, the metrology results can be unreliable due to projection effects. In STEM mode, the Convergent Beam Electron Diffraction (CBED) image is analyzed, via FCN, to determine the specimen tilt orientation, which is then aligned by the microscope stage tilt. With this new analytical technique, the zone axis is aligned to be better than 0.5 deg from zone axis tilt, enabling imaging of silicon dumbbells in the crystal lattice.

CBED analysis can perform tilt alignment in STEM mode without changing any of the optics to acquire a Parallel Beam Electron Diffraction Pattern (DP) image. The clear benefit to this is the ability to perform zone axis alignments at the spot size of the converged beam and very near to the area of interest; this is very useful for lamella with undulations.

The Smart Automation workflow is enabled by machine learning to acquire high resolution images with better than $1 \AA$ resolution. An example image acquired with the automated workflow is presented in Figure 2 with the lattice and FFT corresponding to $0.96 \AA$ resolution. It is through the flexibility of the FCN that Smart Automation can be used for a variety of logic-type semiconductor devices at different points in the manufacturing process. Smart Automation allows a user of any experience level to acquire quality TEM/STEM/EDS data with minimal input. 

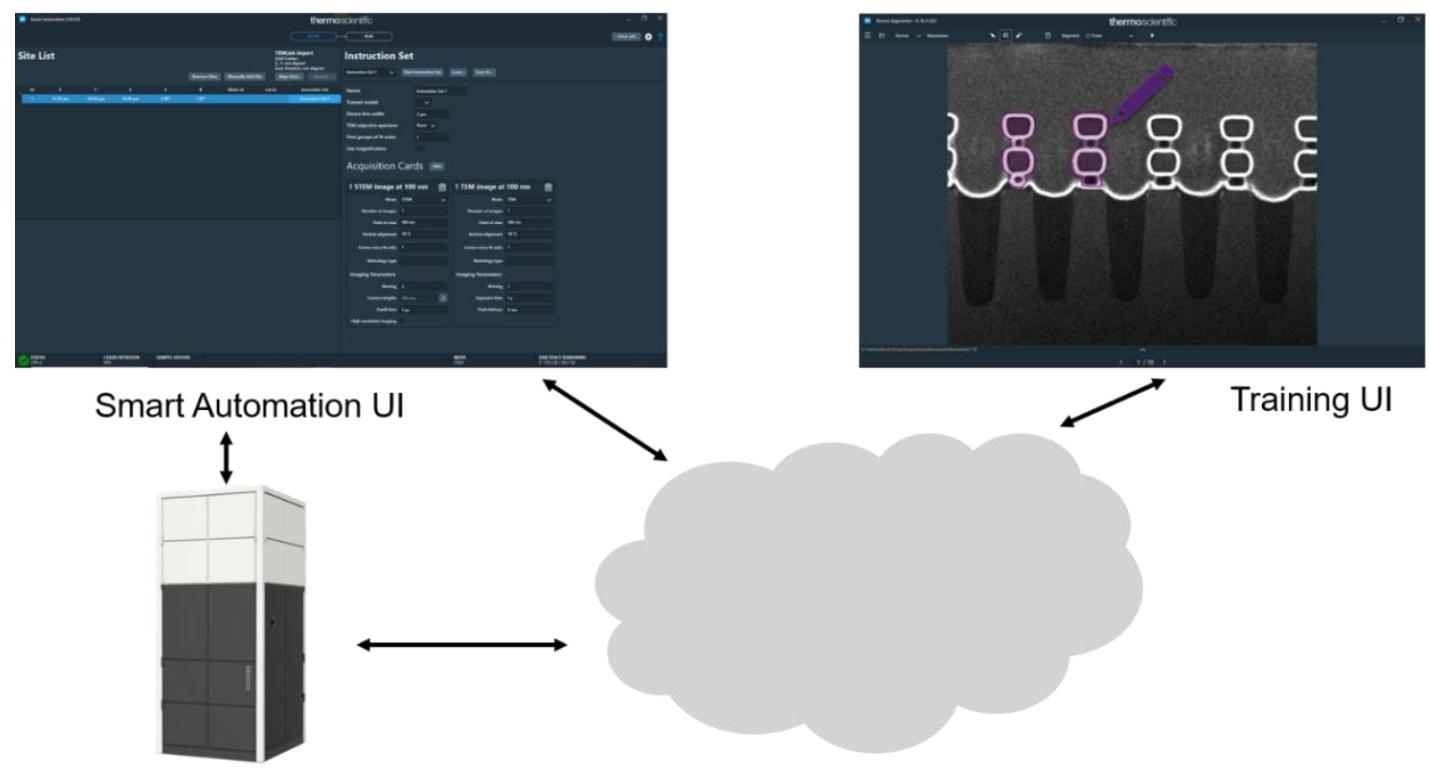

Training UI

Microscope Hardware

Training and Inference Servers

Figure 1. Interaction of FCN training and inference servers with the training UI (Segmenter), the microscope acquisition software (Smart Automation) and the microscope hardware (Metrios)

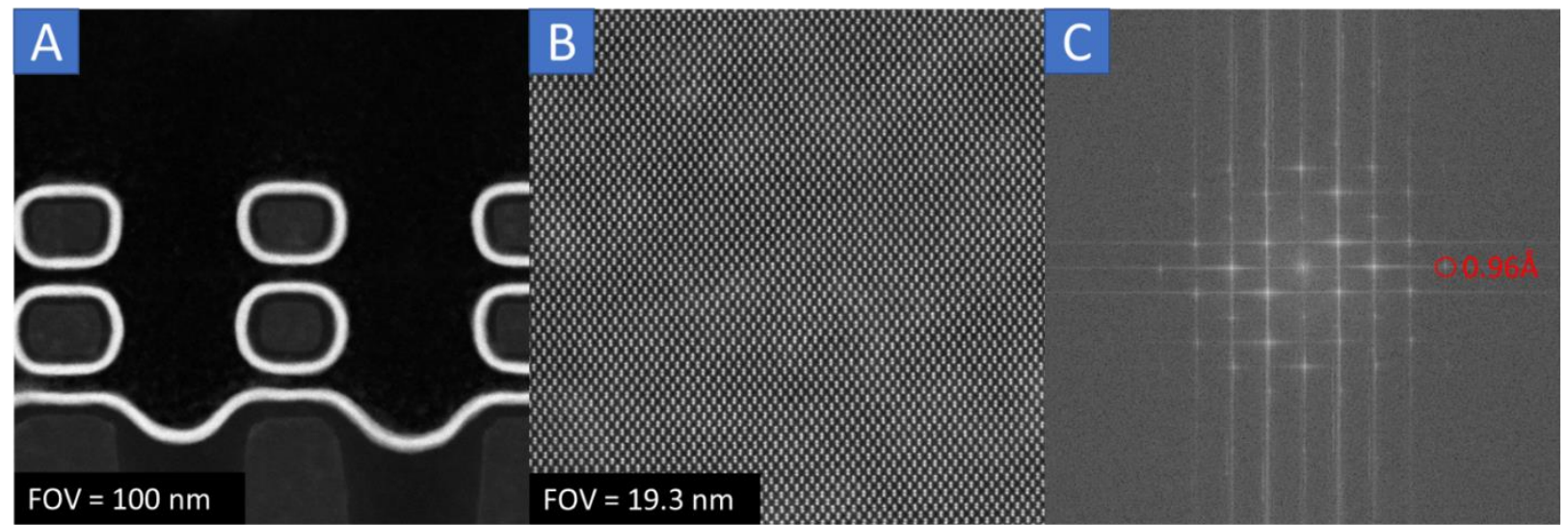

Figure 2. A - Example final STEM HAADF image of an Imec Nanowire at a 100nm FOV acquired with Smart Automation. B - Reference lattice image of Silicon 110 with dumbbell resolution at 19.3nm FOV. C - Reference FFT of lattice image in B highlighting the 3rd order reflections seen with $0.96 \AA$ resolution.

\section{References}

Hayley Johanesen, Michael Strauss, Anne Kenslea, Chris Hakala, Laurens Kwakman, Werner Boullart, Hans Mertens, Yong Kong Siew, and Kathy Barla "Evaluation of the accuracy and precision of STEM and EDS metrology on horizontal GAA nanowire devices", Proc. SPIE 10959, Metrology, Inspection, and Process Control for Microlithography XXXIII, 109591C (26 March 2019); https://doi.org/10.1117/12.2514995

Jonathan Long, Evan Shelhamer, Trevor Darrell. "Fully Convolutional Networks for Semantic Segmentation", Cornell University, Computer Science, Computer Vision and Pattern Recognition, print 1411.4038 (8 March 2015); https://arxiv.org/abs/1411.4038

J. Jansen, M.T. Otten, H.W. Zandbergen, "Towards automatic alignment of a crystalline sample in an electron microscope along a zone axis", Ultramicroscopy, Volume $125 \quad$ (2013), Pages 59-65, ISSN 03043991;https://doi.org/10.1016/j.ultramic.2012.09.010.

(https://www.sciencedirect.com/science/article/pii/S0304399112002458) 